\title{
Visualization of an imploding circular wave front and the formation of a central vertical jet
}

\author{
Amy-Lee Gunter, Othman Smadi, Lyes Kadem and Hoi Dick $\mathrm{Ng}^{\dagger}$ \\ Department of Mechanical and Industrial Engineering, Concordia University, \\ 1455 de Maisonneuve Blvd. West, Montréal, Québec, H3G 1M8, Canada
}

\author{
${ }^{\dagger}$ Corresponding author \\ Department of Mechanical and Industrial Engineering \\ Concordia University \\ 1455 de Maisonneuve Blvd. W. \\ Montréal, QC, H3G 1M8 Canada \\ Tel: (514) 848-2424 ext. 3177 \\ Fax: (514) 848-3175 \\ †e-mail: hoing@encs.concordia.ca
}

Revised short paper submitted to Journal of Visualization

November 2, 2010 


\section{Visualization of an imploding circular wave front and the formation of a central vertical jet}

Amy-Lee Gunter, Othman Smadi, Lyes Kadem and Hoi Dick Ng ${ }^{\dagger}$

Department of Mechanical and Industrial Engineering, Concordia University

Montréal, QC, H3G 1M8 Canada

†e-mail: hoing@encs.concordia.ca

\section{Introduction}

Implosion of surface waves and the resulting formation of a central vertical water jet happen often in nature; they also provide an important analogy for many physical phenomena. For example, when a heavy solid object impacts on a fluid surface, e.g. such as a meteoroid hitting the planet's ocean, a crater is created, which subsequently begins to pinch radially due to the pressure of the surrounding fluid. The collapsing splash results in a high speed upward jet of water (Gekle et al. 2009). The collapse of this jet is perhaps responsible for producing the subsequent principal tsunami (Mader 2004). This implosion phenomenon can also be observed in underwater explosions, where the collapse of a bubble cavity formed at the epicenter of the explosion will result in a high-speed jet, which often results in the damage or destruction of submarines and ships (Blake et al. 1997). In the shallow water limit, the water wave implosion also occurs analogically in nuclear energy fusion where gasdynamic shock waves are imploded to generate dense plasmas of extremely high temperature at the center (Perry and Kantrowitz 1951). In this short paper, the water wave implosion phenomenon is simulated using a simple gate-type experimental setup and the visualization is carried out by video imaging using a high speed camera and by numerical simulation using Volume of Fluid (VOF) method.

\section{Experimental and numerical methods}

The experimental apparatus is shown in Fig. 1. A gate-type water table is constructed $(90 \mathrm{~cm} \times 105 \mathrm{~cm} \times 20 \mathrm{~cm})$ and the creation of a circular converging water wave front is achieved by retracting a $3 \mathrm{~mm}$ thick, $52 \mathrm{~cm}$ in diameter $\left(R_{\mathrm{o}}=\right.$ $26 \mathrm{~cm}$ ) aluminium gate which separates two volumes of water by means of three pneumatic pistons. The table is made of acrylic to permit through-lighting and visualization. A high-speed CMOS camera (pco.1200hs) located at the bottom of the table is used to visualize the dynamics of the implosion at a frame rate of 500 fps. The lighting for the high speed photography is provided by a Pallite VIII system (Cooke, Corp.). The experiments were carried out using tap water with detergent and blue dye added to reduce the surface tension and improve the image contrast. The dynamic viscosity of water is $\mu=1.0 \times 10^{-3} \mathrm{~N} \cdot \mathrm{s} / \mathrm{m}^{2}$ at room temperature of $20^{\circ} \mathrm{C}$. To compare the characteristics of the imploding wave and the occurrence of wave breaking, numerical simulations were carried out with FLUENT software using the Volume of Fluid (VOF) method. One quarter of the complete model was simulated assuming laminar viscous flow with 150,000 elements and $\Delta t=5 \times 10^{-4} \mathrm{sec}$ (Courant Number $\left.C \leq 1\right)$.

\section{Results and discussions}

Figure 2 shows a sequence of snapshots of the implosion process from the video images and simulation results. This result corresponds to an initial water height ratio of 4, with the external and internal water heights of $8 \mathrm{~cm}$ and $2 \mathrm{~cm}$, respectively. Under this condition, a circular water wave can be produced and amplified as it propagates toward the center without wave breaking or strong instability at the circular front. It is due to these physical similarities that an analogy can be drawn between the initial behavior of the shallow water wave and that of a gaseous shock wave, termed the shallow water hydraulic analogy (Kiyanda et al. 2004). It is found however that the region just before implosion is highly unsteady and displays a breaking or tip rolling of the wave front. The wave tends to fold and deform as the top portion of the wave sets water in motion ahead of itself as illustrated in Fig. 3a. Nevertheless the wave focusing still produces a high speed jet (or spout of water) more than $1 \mathrm{~m}$ high at the implosion center. For a direct comparison between results from the experiment and numerical simulation, using Matlab image processing with a simple edge-detection algorithm we systematically track the leading edge of the wave front from the images and estimate correspondingly the averaged wave propagation velocity. As a first step to analyzing the imploding wave, we focus on the rate of implosion (i.e. wave velocity) and the simple front tracking technique used in this study is sufficient. Nevertheless, it is worth noting that advanced techniques are available and capable of analyzing the surface wave topology if the visualization of the complete flow field is desirable (Fouras et al. 2006; Ng et al. 2010). By comparing the velocity of the imploding wave measured from the experiment and the velocity of the simulated wave (see Fig. 3b), there appears to be good agreement within the experimental accuracy and the discrepancy can be attributed to the uncertainty in defining and measuring the wave front radius from image processing. In detail, radial displacement of the wave front is numerically measured at higher resolution with a smaller time interval; the wave shape can be seen more clearly and defined from the simulations at different orientations. Consequently due to the sensitivity to the tracking technique and the use of first-order difference for the velocity computation, a velocity fluctuation resulted in the numerical curve.

Acknowledgment This work is supported by the Natural Sciences and Engineering Research Council of Canada (NSERC). We would like to thank C.B. Kiyanda for providing many useful suggestions and comments on this work. 


\section{References}

Blake JR, Hooton MC, Robinson PB and Tong RP (1997) Collapsing cavities, toroidal bubbles and jet impact. Phil. Trans. R. Soc. Lond. A. 355:537-550.

Gekle S, Gordillo JM, Van der Meer D and Lohse D (2009) Supersonic air flow due to solid-liquid impact. Phys. Rev. Lett. 102:034502.

Kiyanda CB, Chaput P, Higgins AJ and Lee JHS (2004) Investigation of imploding shock waves using hydraulic analogy. Proc. 24th Int. Symp. on Shock Waves, 745-750.

Mader CL (2004) Numerical modeling of water waves, $2^{\text {nd }}$ ed., CRC Press.

Perry WR and Kantrowitz A (1951) The production and stability of converging shock waves. J. Appl. Phys. 22:878886.

Fouras A, Hourigan K, Kawahashi M and Hirahara H (2006) An improved, free surface, topographic technique. J. Vis. 9(1): 49-56.

Ng I, Kumar V, Sheard GJ, Hourigan K and Fouras A (2010) Surface topography of jet shock cells in a hydraulic analogy. J. Vis. 13(1) 175-176. 

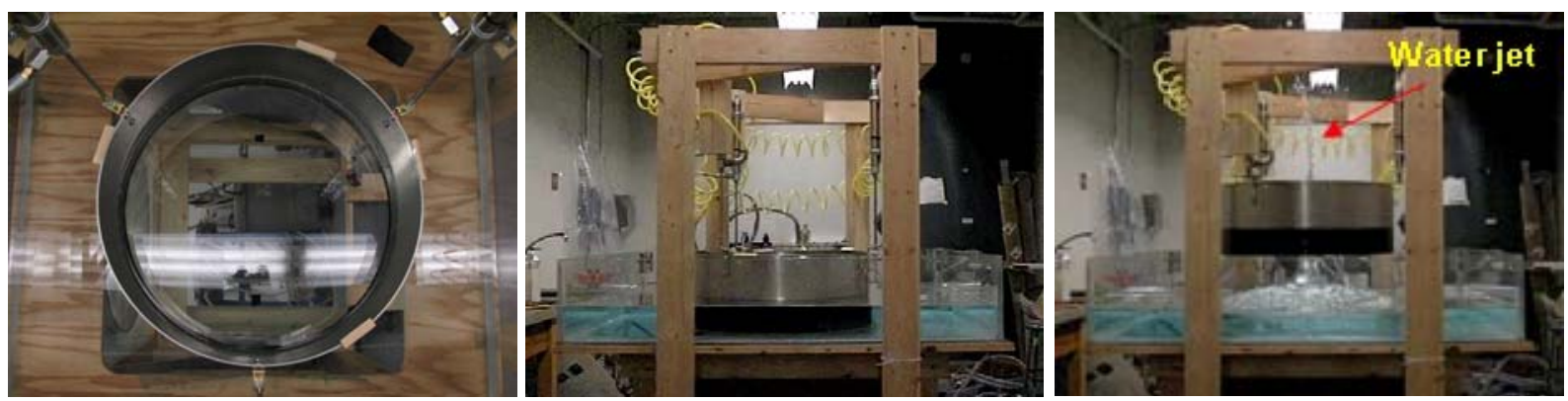

Fig. 1. Experimental setup; Left) Top view of the water table with the circular aluminum gate of radius $R_{0}=26 \mathrm{~cm}$ initially at a closed position; Middle) Side view of the water table (Dimension: $90 \mathrm{~cm} \mathrm{x} 105 \mathrm{~cm} \mathrm{x} 20 \mathrm{~cm}$ ) with the gate initially at a closed position separated two volumes of water; Right) The formation of the central water jet after the extraction of the gate activated by the three pneumatic pistons. 

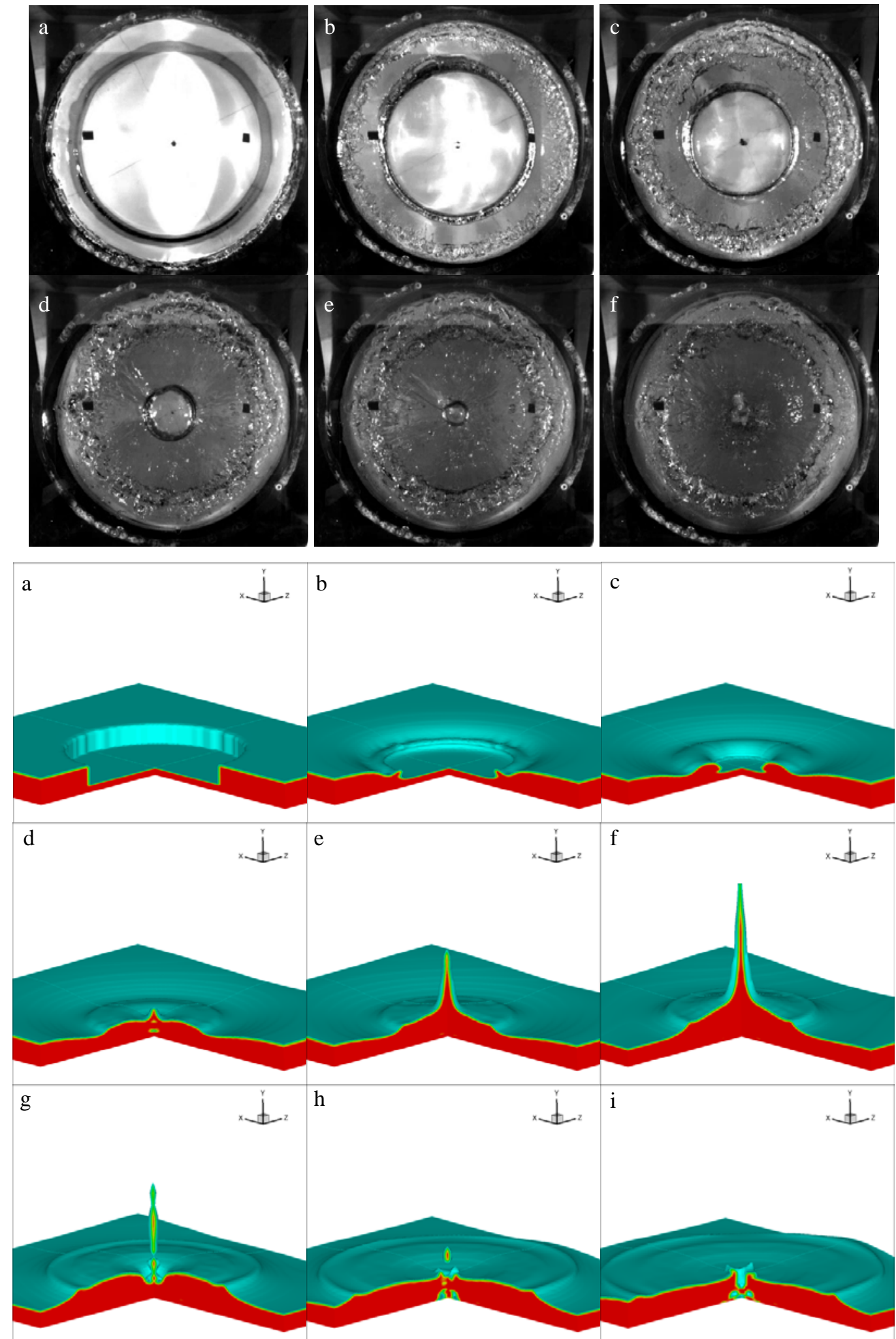

Figure 2. Sequence of different snapshots showing the water wave implosion process. Top) Experimental results; Time displayed are a) 0.05, b) 0.15, c) 0.20, d) 0.30, e) 0.35 and f) 0.40 s. Bottom) Numerical simulation using VOF method; Time displayed are a) 0.05 , b) 0.10, c) 0.20 , d) 0.25 , e) 0.35 , f) 0.45 , g) 0.65 , h) 0.75 , and i) $0.80 \mathrm{~s}$; a)-f) illustrates the implosion sequence as in the experiment; and g)-i) the collapse of the jet. 


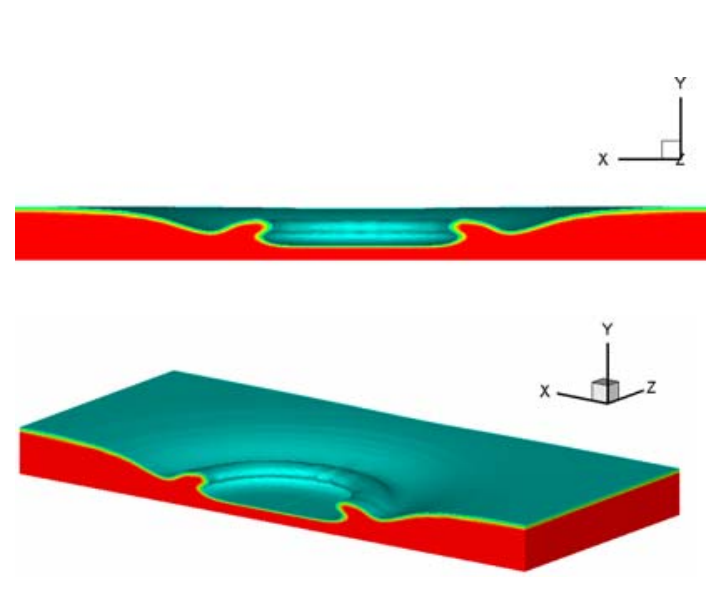

(a)

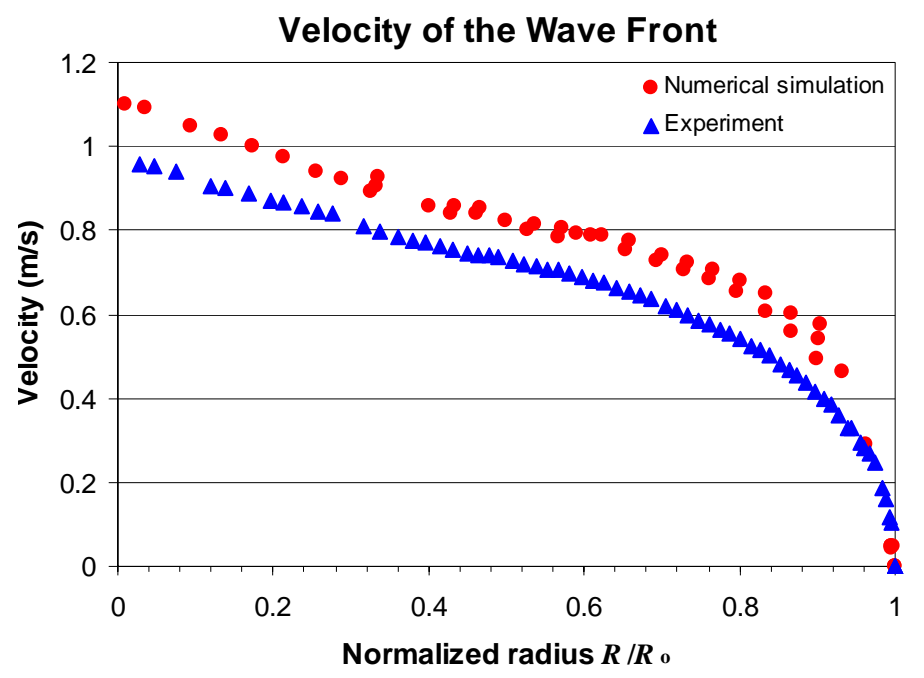

(b)

Figure 3. a) Numerical result showing the rolling of the hydraulic jump; and b) Comparison of the wave velocity between experimental measurement and numerical simulation. 